\title{
Wound Management of Multi-Site Pressure Ulcer at Different Stages in Elderly Patients
}

\author{
Shunqing Su' \\ Xiumei Ding' \\ Huijuan Zou' \\ Yuechun Lin' \\ Jianmin Huang' \\ Disheng Xiong' \\ Jinan Kuan' \\ Yanhong Zhang ${ }^{2}$ \\ Rurong $\mathrm{Xie}^{2}$ \\ 'Department of Burn\&Plast, Dalang \\ Hospital of Dongguan, Dongguang, \\ Guangdong, 523780, People's Republic of \\ China; ${ }^{2}$ Department of Wound Repair, \\ Hongmei Hospital of Dongguan, \\ Dongguang, Guangdong, 523160, People's \\ Republic of China
}

Objective: The present study aims to explore the individualized treatment options for multisite pressure ulcer (PU) at various stages in elderly patients with multiple medical conditions.

Methods: Stages 1 and 2 PU at 146 sites were treated with closed negative pressure suction combined with continuous micro-oxygen perfusion and the local application of foam dressings, silver ion dressings, and moist burn cream. Stages 3 and 4 PU in the sacrococcygeal region were treated with skin or myocutaneous flap transplantation.

Results: Stages 1 and 2 PU healed after treatment with closed negative pressure suction combined with continuous micro-oxygen perfusion and dressing changes. One case died during hospitalization due to an illness. Skin or myocutaneous flap repair was conducted in 34 cases of stage 3 or 4 PU in the sacrococcygeal area. Of these cases, 28 achieved primary healing, and 6 required two or three surgeries, 5 of which received micro-skin implantation. In addition, 10 small deep PU at other sites were repaired by direct excision and suturing or local flap repair. Seven cases were transferred to other departments or hospitals due to concomitant diseases or were discharged automatically without surgical treatment.

Conclusion: Home care for geriatric patients is difficult. PU often occur at multiple sites because of the duration of various pressures, and different sites may demonstrate different stages because of varying degrees of pressure. When actively treating stages 3 and $4 \mathrm{PU}$, the trauma management of stages 1 and 2 PU should not be neglected.

Keywords: oldest-old patients, pressure ulcer, wound management, flap transplantation, micro-skin implantation

\section{Introduction}

A pressure ulcer (PU) is an ischemic necrosis of the skin and deep tissues caused by long-term pressure on local tissues, impaired blood circulation, tissue ischemia, and hypoxia to the point of necrosis. ${ }^{1}$ With the development of the economy and the improvement in the quality of life, China has gradually started to become an aging society, and the incidence of PU is positively correlated with age. Previous study suggested that older people were more likely to develop PU. ${ }^{2}$ As the elderly suffer from chronic diseases such as diabetes mellitus, tumors, and cardiovascular and cerebrovascular diseases, they are often unable to care for themselves. It is difficult for them to turn over on their own, and when they stay in one position for extended periods of time, PU may form at multiple sites either simultaneously or successively. Once they have formed, PU are difficult to treat and cause great distress to patients and their families. In addition, a variety of medical conditions further complicate treatment. ${ }^{3,4}$ Over the past five years, 52 elderly patients with
Correspondence: Xiumei Ding Department of Burn\&Plast, Dalang Hospital of Dongguan, No. 85 of Jinlangzhong Street, Dalang District, Dongguang, 523780, People's Republic of China

Tel $+86769-83009512$

Fax $+86769-83033122$

Email ding_xm123@outlook.com 
sacrococcygeal PU were admitted to our hospital, and the clinical data were summarized in order to discuss the treatment methods for PU in different stages at various sites.

\section{Methods}

In the present study, a total of 52 elderly patients with complaints of PU in the sacrococcygeal region of the buttocks from January 2016 to October 2020 were enrolled. Staging of the pressure ulcer: according to the histopathological changes, ${ }^{1} 43$ stage 1 lesions were confined to the epidermis and dermis, and the lesions were mostly located in a pressure area outside of the sacrococcygeal region. There were 103 stage 2 PU that were beyond the dermis, and 17 stage 3 PU had caused damage to the depth of the subcutaneous adipose tissue including 10 cases in the sacrococcygeal region and 7 in other regions. Of the stage 4 cases, 39 were in the sacrococcygeal region, and 3 were in other regions with deep trauma to the muscular layer of the bone. All cases were accompanied by multiple diseases including 26 cases with diabetes mellitus, 20 with hypertension, 19 with cerebrovascular disease, and 3 with fractures. The largest number of concomitant diseases was 12 , and the average was $4-5$.

\section{Therapeutic Methods}

Stage 1 PU manifested as red skin and epidermal erosion and were treated with self-adhesive foam dressings, ulcer patches, and airbed sponge pads for pressure reduction. The lesions were treated by releasing local pressure and changing the dressing to an ampoule patch, foam dressing, and moist burn cream. Stage 2 PU manifested as a skin rupture not reaching the whole skin layer with exudate and blisters and were treated with continuous micro-oxygen infusion combined with closed negative pressure suction. ${ }^{5}$ Gradual healing was achieved by changing the dressing to a silver ion dressing and using moist burn cream. Red-light local irradiation could accelerate wound healing.

In patients with stage 3 or $4 \mathrm{PU}$, there was often massive necrosis of the deep and superficial fascia and muscles; separation of the subcutaneous tissues from the muscles; formation of thick hard scabs of necrotic skin and subcutaneous tissues, septic infection under the scabs, and subterranean sinus tracts to the periphery of the wound; massive necrotic tissues; and sepsis, and some of these ulcers were accompanied by generalized hyperthermia. Thorough removal of scabs and attached necrotic tissues, the use of hydrocolloid dressing changes ${ }^{6}$ could better control the local infection before surgery, and closed negative pressure drainage were used. When the granulations grew well, flap transfer repair was performed, in which the muscle flap of the gluteus maximus was transferred to cover the exposed area of the sacral bone and was covered with the superior gluteal artery perforated flap. A doublelumen drainage tube was placed under the flap and in the operated area for closed negative pressure treatment. In most of the patients, the second surgery failed because the base and the flap could not adhere to each other, and these patients were treated with micro-skin implantation and epithelial crawling. For stage 3 PU, in which the flap portion was small and the depth was limited to the full skin layer, the patients were treated with closed negative pressure suction combined with continuous micro-oxygen perfusion, and in some cases, the lesions healed with dressing changes and moist burn cream exposure therapy (Table 1).

\section{Results}

The patients consisted of 18 males and 34 females aged 62-93 years including 10 aged 62-69 years and 42 aged 70-93 years. There were 52 cases with PU in the sacrococcygeal region and 43 with PU in two or more sites (up to 10 sites). In all cases, except for PU in the sacrococcygeal region, most of the PU were at different stages at

Table I Stages and Treatment Options $(n=205)$

\begin{tabular}{|l|l|l|l|l|l|l|l|}
\hline \multirow{2}{*}{ Stage } & \multicolumn{4}{l}{ Nonoperative Treatment } & \multicolumn{2}{l|}{ Surgical Treatment } \\
\cline { 2 - 7 } & NPS & NPS+CMOI & FD/ SID & TCM & Excision and Suture & Flap Transfer & Micro-Skin Implantation \\
\hline I & 0 & 0 & 43 & 0 & 0 & 0 & 0 \\
II & 3 I & 26 & 28 & 18 & 0 & 0 & 0 \\
III & 3 & 8 & 0 & 0 & 16 & 0 & 0 \\
IV & 0 & 0 & 0 & 0 & 3 & 34 & 5 \\
\hline
\end{tabular}

Abbreviations: NPS, negative pressure suction; CMOI, continuous micro-oxygen infusion; FD, foam dressing; SID, silver ion dressing; TCM, Traditional Chinese medicine. 
Table 2 Occurrence Sites and Stages Summary of Pressure Ulcer $(n=205)$

\begin{tabular}{|l|l|l|l|l|}
\hline Occurrence Site & I & II & III & IV \\
\hline Sacrococcygeal & 0 & 3 & 9 & 39 \\
Heel & 10 & 24 & 4 & 2 \\
Lateral malleolus & 13 & 35 & 2 & I \\
Iliac crest & 8 & 22 & I & 0 \\
Elbow joint & 5 & 8 & 0 & 0 \\
Scapula and other site & 7 & II & I & 0 \\
\hline
\end{tabular}

three to five sites. The occurrence sites and stages summary of pressure ulcer were summarized in Table 2.

Flap or myocutaneous flap repair was conducted in 34 cases of stage 3 or $4 \mathrm{PU}$ in the sacrococcygeal area. Of these, 28 achieved primary healing (Figures 1 and 2), 6 required two or three surgeries, 5 of which received micro-skin implantation (Figures 3 and 4), and 10 small deep PU in other sites were repaired by direct excision and suturing or local flap repair. Seven cases were transferred to other departments or hospitals due to concomitant diseases or were discharged automatically without surgical treatment. At other sites, 146

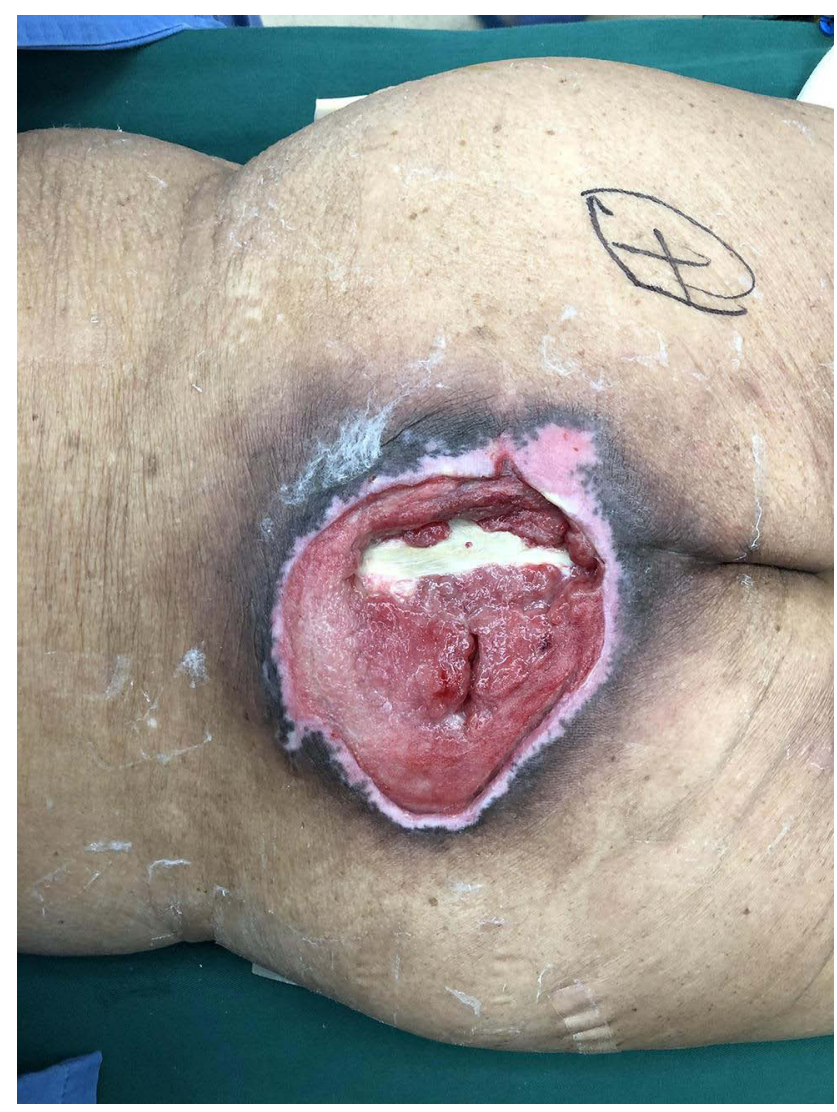

Figure I Preoperative condition in Case I: Stage 4 sacrococcygeal pressure ulcer $(9.2 \times 9 \mathrm{~cm})$ with one-stage repair after flap grafting.

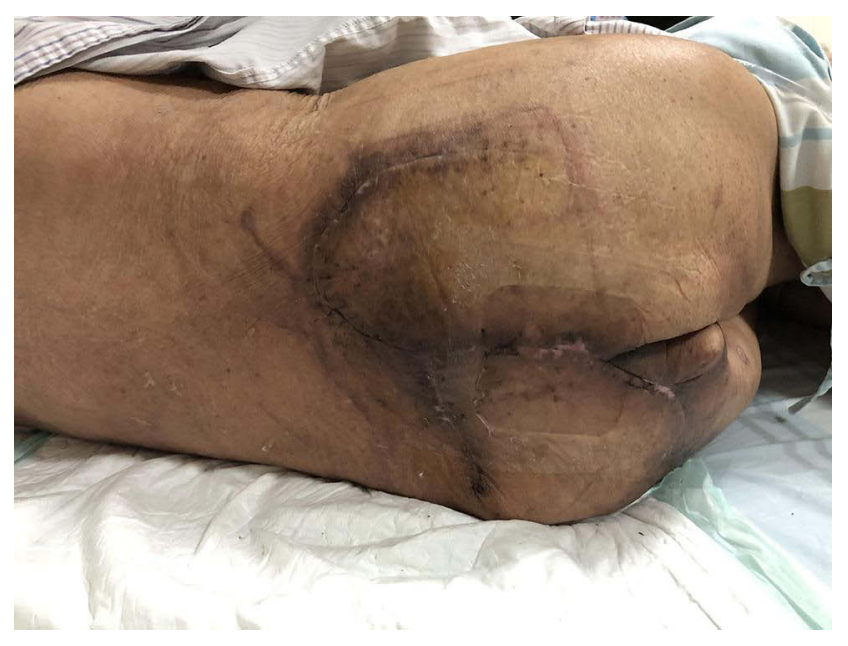

Figure 2 One month after grafting in Case I, with good flap survival.

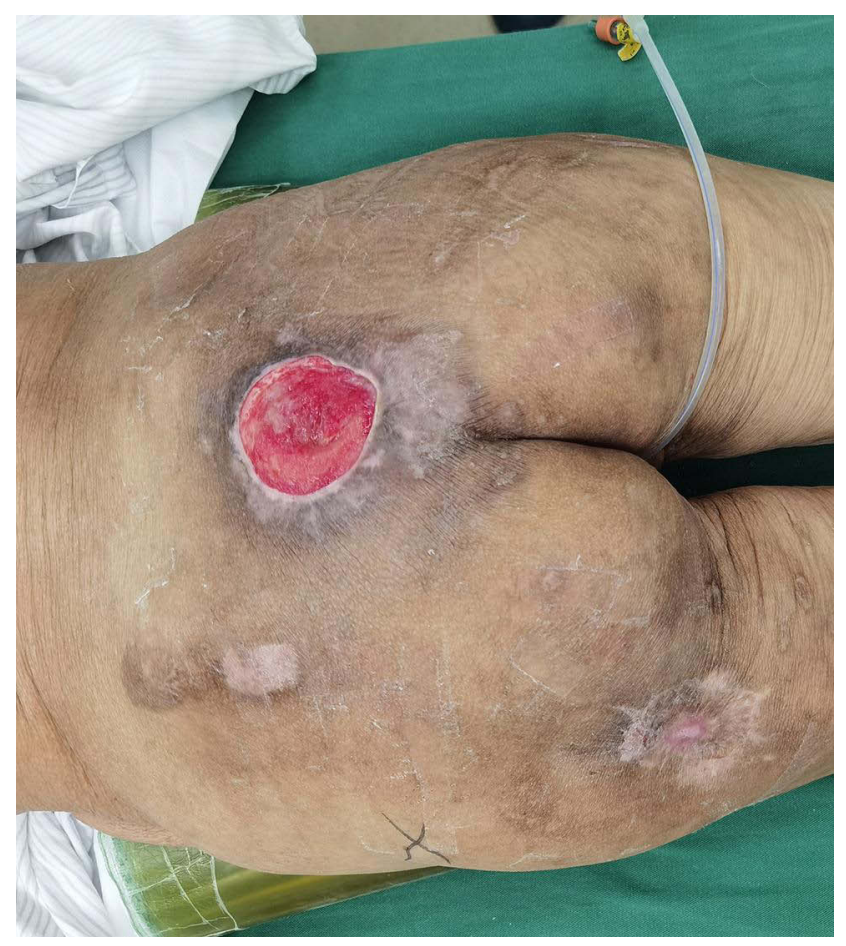

Figure 3 Preoperative condition in Case 2: Sacrococcygeal pressure ulcer $(6 \times 7 \mathrm{~cm})$ with failure of the wound healing after flap grafting and conduction of the second phase micro-skin implantation.

stage 1 or $2 \mathrm{PU}$ were present and were treated with closed negative pressure suction combined with continuous microoxygen infusion and healed after dressing changes. One case died during hospitalization due to an illness.

Mantel-Haenszel chi-square test results indicated that there was a linear trend between pressure ulcer stage and therapeutic effect, $\chi^{2}=32.86, P<0.001$. Furthermore, the effect decreases as the stage of pressure ulcer increases (Table 3). 


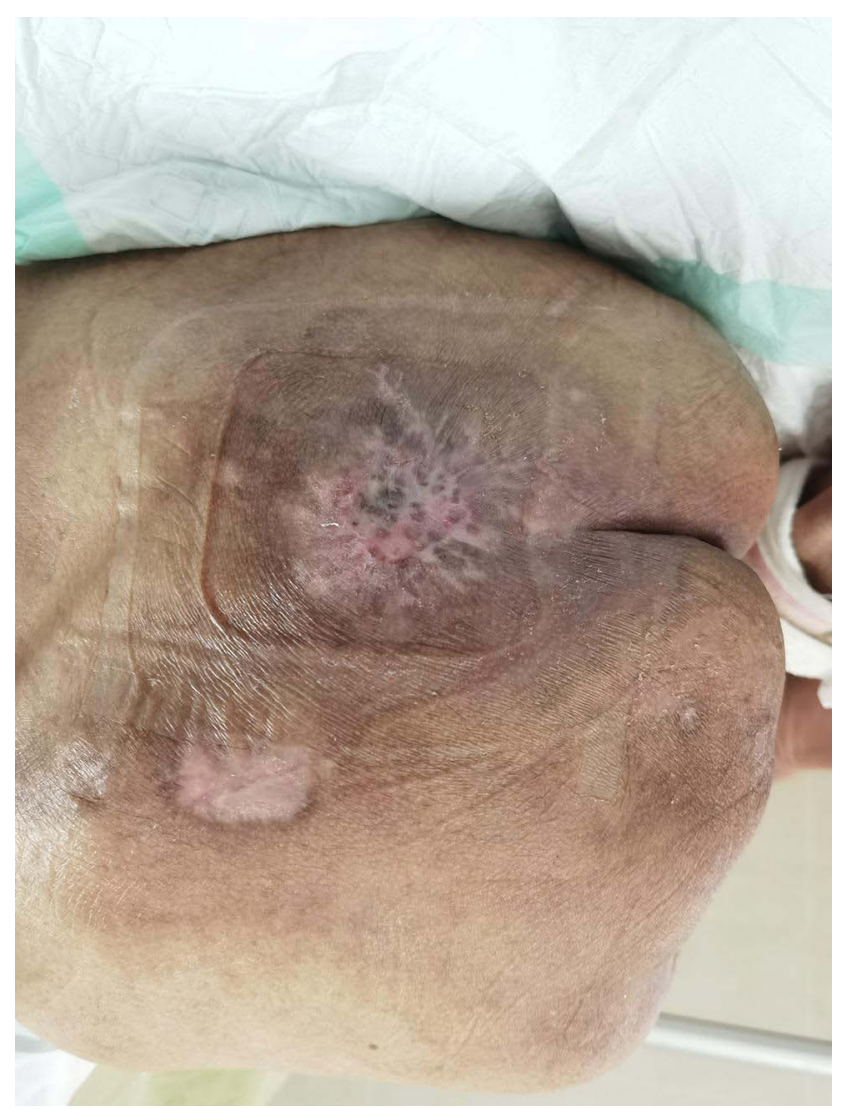

Figure 430 days after the operation with wound healing in Case 2.

\section{Discussion}

The thin, dry skin and atrophied subcutaneous fat in the elderly make the compression area vulnerable to pressure due to insufficient cushioning, while the poor nutritional status and poor healing ability of wounds make PU difficult to treat over time. ${ }^{7} \mathrm{PU}$ are mainly formed in the sacrococcygeal region, bilateral hips, heels, ankle joints, and other areas of the body experiencing high pressure. These areas have no muscle wrap or a thin muscle layer, lack tissue protection, and are often compressed. In the supine position, the most common places for PU are the sacrococcyx and the heel. In the lateral position, PU are prone to occur in the greater trochanter, the lateral ankle, and other parts. When sitting,

Table 3 Stages and Treatment Outcomes (n, \%)

\begin{tabular}{|l|l|l|l|l|}
\hline \multirow{2}{*}{ Stages } & \multirow{2}{*}{ Number of PU } & \multicolumn{3}{|l|}{ Treatment Outcomes } \\
\cline { 3 - 5 } & & Cured & Improved & Failure \\
\hline I and II & 146 & $140(95.89)$ & $6(4 . I 1)$ & 0 \\
III & I7 & $14(82.35)$ & $3(17.44)$ & 0 \\
IV & 42 & $28(66.67)$ & $6(14.28)$ & $7(16.67)$ \\
\hline
\end{tabular}

Abbreviation: PU, pressure ulcer. the most common place for PU is the ischial tuberosity. Medical conditions such as anemia, hypoproteinemia, and diabetic peripheral neuropathy that accompany PU in the elderly contribute to the difficulty in treating PU. In elderly patients on long-term hormone medication, the thinning of the skin and the difficulty in controlling traumatic infections due to immunosuppression are also correlated with difficulty in treating PU.

Older patients with multiple medical conditions are difficult to care for at home because both the force and duration of pressure vary, and PU often occur in different stages at multiple sites due to different pressure exerted at various sites. Treatment often focuses on severe stage 3 and 4 PU, while ignoring stage 1 and $2 \mathrm{PU}$ in other areas, resulting in these deepening to stage 3 or 4 over time. The present study focused on the surgical treatment of stage 3 and 4 PU in the sacrococcygeal region, while not neglecting the trauma treatment of stage 1 and 2 PU in other areas in order to avoid progression to deep PU. Our treatment achieved simultaneous recovery and shortened the average length of stay in hospital.

Patients with stage 3 and 4 PU have more and deeper necrotic tissue, and surgery is the most rapid and effective means of treatment ${ }^{8,9}$ by debridement and irrigation using an ultrasonic debridement machine, closed negative pressure drainage of the wound, and fiber silver dressing filling for those with a submerged sinus. Surgical treatment is possible when the granulation is fresh with complete excision of the scar tissue around the pressure ulcer and the aging inwardly growing epithelial tissue together with the complete removal of the basal fibrous plate and necrotic tissue from the inner wall of the sinus and eradication of the exposed bone cortex. ${ }^{10}$ Repeated rinsing with hydrogen peroxide and normal saline should be conducted with complete hemostasis. For sacrococcygeal PU, the superior and inferior gluteal artery perforated flap was designed according to the size of the wound and the cavity; bone exposures were filled with the muscle flap ${ }^{11}$ and sutured layer by layer without leaving a dead cavity; and a negative pressure drainage tube was placed under the flap or muscle flap and on the flap surface to conduct the double-lumen drainage. In five cases, the basal fiber plate was too thick to adhere closely to the flap resulting in a subterranean cavity under the flap after surgery, which made it difficult to transplant the flap again. In these cases, the wound was closed mainly by using micro-ski dotted implantation. ${ }^{12}$ The disadvantage of this method is that the new epidermis may not be resistant to wear, and care should be well conducted postoperatively and at home. 
This study has the following limitations. Firstly, the number of patients is small, and there is a lack of a larger sample size for statistical testing. Secondly, this study is a retrospective study, which can only reflect the efficacy of a single center. Further study, such as random control trial, should be carried out to better evaluate the efficacy.

The treatment of PU in elderly patients with multiple medical conditions is a multidisciplinary and comprehensive treatment process, and it is essential to establish a multidisciplinary team. It is necessary to treat the primary disease, correct anemia and hypoproteinemia promptly, control blood glucose and blood pressure within normal ranges, and create good conditions for surgery. While actively making preoperative preparations for the stage 3 and $4 \mathrm{PU}$, conservative treatment of stage 1 and 2 $\mathrm{PU}$ in other areas should not be neglected.

\section{Ethics Approval and Consent to Participate}

This study was conducted with approval from the Ethics Committee Dalang Hospital of Dongguan. This study was conducted in accordance with the declaration of Helsinki. Written informed consent was obtained from all participants.

\section{Consent for Publication}

All patient guardians signed a document of informed consent.

\section{Acknowledgments}

We would like to acknowledge the hard and dedicated work of all the staff that implemented the intervention and evaluation components of the study.

\section{Funding}

Project approved by Dongguan Science and Technology Bureau (201950715040143).

\section{Disclosure}

The authors declare that they have no competing interests.

\section{References}

1. Kottner J, Cuddigan J, Carville K, et al. Prevention and treatment of pressure ulcers/injuries: the protocol for the second update of the international clinical practice guideline 2019. J Tissue Viability. 2019;28(2):51-58. doi:10.1016/j.jtv.2019.01.001

2. Børsting TE, Tvedt CR, Skogestad IJ, et al. Prevalence of pressure ulcer and associated risk factors in middle- and older-aged medical inpatients in Norway. J Clin Nurs. 2018;27(3-4):e535-e543. doi:10. 1111/jocn. 14088

3. Xing YC, Zhao SH, Huo LL. Discussion the evaluation occasion and nursing intervention of pressure sores risk factor for elder patient. Nurs Pract Res. 2011;8(4):26-27.

4. Jordan SW, De la Garza M, Lewis VL Jr. Two stage treatment of ischial pressure ulcers in spinal cord injury patients: technique and outcomes over 8 years. J Plast Reconstr Aesthet Surg. 2017;70 (7):959-996. doi:10.1016/j.bjps.2017.01.004

5. Li YZ, Hu XD, Li YF, et al. Influence of negative pressure-assisted wound therapy combined with topical oxygen therapy on the healing of wound surface of rabbits. J Trauma Surg. 2015;17(1):9-11.

6. Wang WH, Han XM, Ji XH, et al. Clinical effect of hydrocolloid dressings on treatment of pressure ulcer children complicated with moderate and severe infections. Chin J Nosocomiol. 2019;29 (06):949-952.

7. Shahin ES, Dassen T, Halfens RJ. Pressure ulcer preva - lence and incidence in intensive care patients: alitera - ture review. Nurs Crit Care. 2008;13(2):71-79.

8. Nrezgoda JA, Mendez - Estman S. The effective management of pressure ulcers. Adv Skin Wound Care. 2006;19(Suppl 1):3-15. doi:10.1097/00129334-200601001-00001

9. Lin CT, Chang SC, Chen SG, et al. The superior gluteal artery perforator flap for reconstructive of sacral sores. Saudi Med J. 2016;37(10):1140-1143. doi:10.15537/smj.2016.10.15682

10. Lin C-T, Chen S-Y, Chen S-G, et al. Parasacral perforator flaps for reconstruction of sacral pressure sores. Ann Plast Surg. 2015;75 (1):62-65. doi:10.1097/SAP.0000000000000024

11. Kim CM, Yun IS, Lee DW, et al. Treatment of ischial pressure sores with both profunda femoris artery perforator flaps and muscle flaps. Arch Plast Surg. 2014;41(4):387-393. doi:10.5999/aps.2014.41.4.387

12. Qin GQ, Ding MH. Clinical experience of treating granulation wounds at the late stage of PU with a combination therapy. Chin J Burns Wound Surface Ulcer. 2019;31(4):257-260.
Clinical, Cosmetic and Investigational Dermatology is an international, peer-reviewed, open access, online journal that focuses on the latest clinical and experimental research in all aspects of skin disease and cosmetic interventions. This journal is indexed on CAS.
The manuscript management system is completely online and includes a very quick and fair peer-review system, which is all easy to use. Visit http://www.dovepress.com/testimonials.php to read real quotes from published authors. 